Sarah Marinucci und Marie Urban: Freie Republik HORA Regie und Schauspiel als fortwährender Aushandlungsprozess. Ein Gespräch mit Gianni Blumer (Schauspieler Theater HORA) und Nele Jahnke (Stv. künstlerische Leiterin Theater HORA). In: Publikum im Gegenwartstheater. Hg. v. Beate HochholdingerReiterer, Géraldine Boesch, Marcel Behn. Berlin: Alexander 2018 (itw : im dialog 3), S. 216-223. https://doi.org/10.16905/itwid.2018.19 


\section{Freie Republik HORA - Regie und Schauspiel als fortwährender Aushandlungsprozess}

Ein Gespräch mit Gianni Blumer (Schauspieler Theater HORA) und Nele Jahnke (Stv. künstlerische Leiterin Theater HORA).

Das Gespräch wurde in Schweizerdeutsch und Hochdeutsch geführt. Für die Publikation wurde die Sprache vereinheitlicht.

Gott ist der vierte Teil des Langzeitprojekts Freie Republik HORA einer Produktionsplattform von Theater HORA, die sich als künstlerisch-soziales Labor versteht. In diesem Rahmen führen professionelle Schauspieler_innen mit einer >geistigen Behinderung`seit 2013 in wechselnden Teams kollektiv Regie. So hinterfragt auch Gott jegliche Form von Alleinherrschaftsanspruch und wagt sich an ein Genesis-Experiment, welches nichts Geringeres als die Neuerschaffung der Welt zum Ziel hat. Strukturiert ist die stetig im Wandel begriffene Inszenierung durch die sieben Tage der biblischen Schöpfungsgeschichte.

Sarah Marinucci: Theater HORA aus Zürich ist das einzige professionelle Theater der Schweiz, dessen Schauspieler_innen alle eine IVzertifizierte `geistige Behinderung \ haben. Neben seinen wechselnden Zusammenarbeiten mit nicht-behinderten Regisseur_innen und Choreograf_innen, startete Theater HORA im Jahr 2013 das auf fünf Jahre angelegte Langzeitprojekt und Theaterexperiment Freie Republik HORA. In mehreren Schritten geht es in diesem prozessorientierten und mehrere Phasen durchlaufenden Projekt darum, dass die Ensemblemitglieder von Theater HORA selbst Regieprojekte entwickeln. In 
Phase Eins erarbeiten die Mitglieder von Theater HORA gemeinsam eine Inszenierung in kollektiver Regie ohne die künstlerische Mitsprache von Michael Elber und Nele Jahnke, der künstlerischen Leitung von Theater HORA. Anschließend werden in der zweiten Phase die ersten Eigenregie-Versuche unternommen, wobei die Ensemblemitglieder innerhalb weniger Tage verschiedene kleinere Regiearbeiten zu selbst ausgesuchten Themen erarbeiten, mit denen sie sich intensiv beschäftigen wollen und die dann in Form von Tryouts vor Publikum aufgeführt werden. ${ }^{1}$ In der dritten Phase entwickeln die Ensemblemitglieder je ein individuelles Regiekonzept, das einer externen Fachjury zugeschickt wird.

Die drei Phasen wurden im Sommer 2016 als eine Retrospektive der dritten Phase abgeschlossen, was uns zur vierten Phase und somit zur Inszenierung Gott führt, die wir gestern Abend miterleben durften. ${ }^{2}$ Nele Jahnke, können Sie uns erzählen, wie sich die Idee für diese vierte Phase aus den vorangehenden Phasen entwickelt hat?

Nele Jahnke: Die im letzten Jahr abgeschlossene dritte Phase von Freie Republik HORA beinhaltete sechs Regiearbeiten von sechs HORARegisseur_innen, deren Konzeptideen von einer externen Fachjury ausgewählt wurden. Die Regisseur_innen erarbeiteten mit einem fixen Budget und teilweise unter Beteiligung externer Künstler_innen sechs eigene Regieprojekte. Eines der sechs Regiekonzepte stammte von Gianni Blumer. Bis einschließlich zur dritten Phase übernahm die künstlerische Leitung von Theater HORA, sprich Michael Elber und ich, die Rolle der Assistenz. Wir haben diesbezüglich die Regel aufgestellt, uns in den künstlerischen Produktionsprozess nicht einzumischen, sofern die HORA-Künstler_innen sich an das Regelwerk von Freie Republik HORA und die darin festgelegten fünf Bedingungen hielten. Die erste Bedingung besagt, dass alles, was nicht verboten ist, erlaubt ist. Verboten sind sexuelle Übergriffe, Gewalt und Zerstörung fremden Eigentums. Die zweite Bedingung hält fest, dass jede_r Regisseur_in eine Woche Probenzeit erhält, wobei die Probebühne zur freien Verfügung steht. Als dritte Bedingung muss jede_r Regisseur_in vor Probenbeginn einen Titel genannt und ein Inszenierungskonzept erstellt haben. Die vierte Bedingung lautet, dass ein 
Produktionsbudget von maximal 4.00o CHF Gage und 1.00o CHF Produktionsaufwendung pro Regisseur_in und Woche für eine von ihr oder ihm bestimmte Anzahl von maximal drei externen Künstler_innen zur Verfügung steht. Die fünfte und letzte Bedingung ist schließlich eine individuelle Bedingung, die auf das künstlerische Profil der einzelnen Regisseur_innen zugeschnitten ist und von der künstlerischen Leitung bestimmt wird. Doch Michael Elber und ich hatten im Laufe dieser Phase immer mehr das Bedürfnis, wieder intensiver am künstlerischen Produktionsprozess mitzuwirken, was wir in den ersten drei Phasen bewusst nicht gemacht hatten. Der nächste logische Schritt war, diese vierte Phase und somit die Inszenierung Gott zu realisieren, an welcher alle beteiligt sind, zusammenarbeiten und sowohl in der Regie- als auch in der Schauspielfunktion tätig sein können.

Marie Urban: Hier anknüpfend möchte ich gerne auf Ihre Arbeitsweise eingehen. Bei der Rezeption von Gott wirkten viele Szenen improvisiert. Wie sind diese Szenen entstanden?

Gianni Blumer: Zuallererst haben wir uns alle in einem Kreis zusammengesetzt und uns überlegt, was jede_r von uns möchte. Jede_r hat sich Gedanken zum Thema `Gott ‘ gemacht. Diese Szenen konnten wir dann in das Stück einbauen. Wir haben auch Duo-Regie gemacht. Ich arbeite zum Beispiel mit Noha Badir zusammen. Wir machen diese Arbeit also zu zweit.

Sarah Marinucci: Wurden alle Szenen bereits im Vorfeld festgelegt? Oder gab es auch Elemente, die während der Aufführung im Moment entstanden sind?

Nele Jahnke: Es gibt Szenen, die festgelegt sind, nämlich der Anfang ${ }^{3}$ und die Tage Eins und Zwei sowie der Schluss mit den Tagen Sechs und Sieben. Dazwischen sind die Szenenabläufe der Tage nicht festgelegt. Diese Szenen diskutieren wir vor jeder Aufführung wieder aufs Neue. Uns steht ein Materialpool zur Verfügung, der aus eben den Szenen besteht, die von den - bereits von Gianni Blumer erwähnten - Regie-Duos generiert wurden. Es ist jedoch nicht klar, wer in diesen 


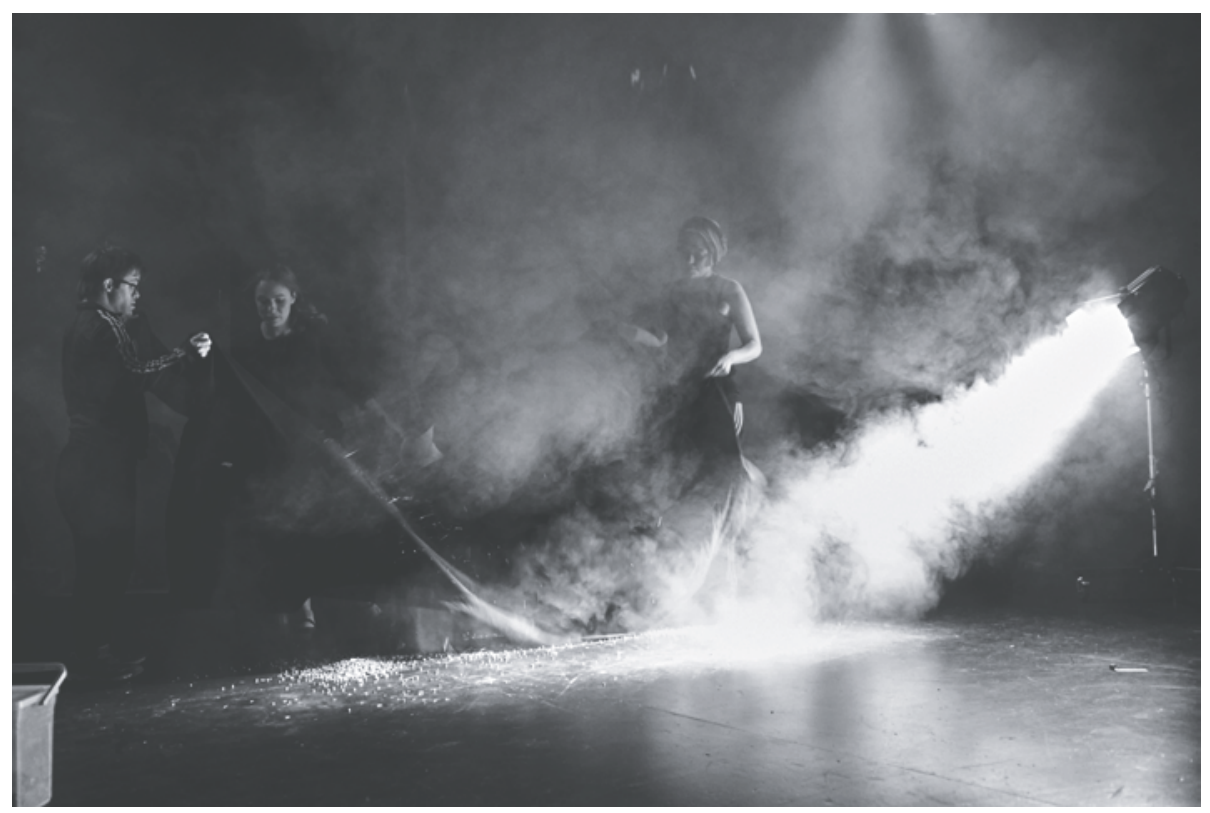

Gott, Theater HORA

Foto: Anita Affentranger

Szenen welche Rolle übernimmt. Weder die Besetzung der Schauspielenden noch diejenige der Regisseurin oder des Regisseurs ist vorbestimmt. Und so geschehen `Zufälligkeiten`. Eine solche Zufälligkeit ist zum Beispiel die Socke von Gianni Blumer, die bei der gestrigen Aufführung einfach nicht mehr auffindbar war.

Gianni Blumer: Meine Socke verschwand während des `Kleiderspiels‘. In dieser Szene führen Remo Beuggert und Nora Tosconi Regie. Nora und ich haben uns ausgezogen, und die anderen hatten die Idee, die Kleider zu verstecken. Es ging darum, wer zuerst seine Kleider wiederfinden und anziehen kann. Nora hat gewonnen, da ich meine Socke nicht mehr gefunden habe. Ich habe gesucht und gesucht, aber ich habe sie während der ganzen Aufführung nicht wiedergefunden. Erst ganz zum Schluss hat sie Nora dann entdeckt.

Marie Urban: Diese kollektive Arbeitsweise ist sehr spannend, weil Sie damit auch die klassische Aufteilung der Regieführung infrage 
stellen. Die Bühne sieht bei Gott aus wie ein Labor, in dem künstlerische Experimente durchgeführt werden. Wie kommt es im Rahmen einer solchen Konstellation dazu, dass jemand einen Vorschlag machen kann, der dann akzeptiert wird? Und wer entscheidet, was akzeptiert wird und was nicht?

Gianni Blumer: Wir probieren einfach immer aus. Es ist immer ein Versuch. Wir haben auch schon mal bei Tag Sieben begonnen und sind dann in dieser Reihenfolge bis zum Anfang gekommen. Die Szenen können auch abgeschnitten oder zerstückelt werden, woraus dann eine neue Reihenfolge entsteht. Was an den Abenden gemacht wird, entscheidet die Gruppe. Vor jeder Aufführung gibt es eine Versammlung im Kreis, und wir sprechen darüber, was wer machen möchte. Die Idee dabei ist, dass der Abend als Chance genutzt werden kann und so die Aufführung gut wird.

Nele Jahnke: Genau, wir machen diese berühmte Versammlung im Kreis. In diesem Rahmen darf jede Person Vorschläge für die noch nicht festgelegten Szenen respektive Tage vorbringen. Im Anschluss daran wird abgestimmt, die Mehrheit gewinnt. Gleichzeitig wird jedoch auch offen manipuliert; ich kann beispielsweise Gianni mit einer Coca Cola bestechen, damit er für meinen Vorschlag stimmt. Außerdem ist Geduld in einem so großen Kollektiv, das gemeinsam Entscheidungen treffen muss, Voraussetzung. Jede_r hat eigene Wege, wie sie oder er damit umgeht. Zusätzlich muss jedoch auch erwähnt werden, dass trotz des Versuchs, eine kollektive Arbeitsweise zu entwickeln, bei welcher die Dramaturgie vom Kollektiv bestimmt wird, Michael Elber und ich stets in einem Widerspruch zu unserer Funktion als künstlerische Leitung stehen, vor allem dann, wenn es um technische Einrichtungen oder sonstige organisatorische Angelegenheiten geht. Da werden gewisse Entscheidungen auch einfach von uns, also von nur einer oder zwei Personen getroffen. Andere dann wiederum im Kreis.

Marie Urban: Wie ist es denn bei Ihnen, Gianni Blumer? Sind Sie in Bezug auf die Inszenierung Gott lieber in der Position der Regie oder in derjenigen, in welcher Sie Anweisungen befolgen müssen? 
Gianni Blumer: Ich mache beides am liebsten.

Sarah Marinucci: Abschließend und bevor wir zum Publikum hin öffnen, möchte ich gerne eine letzte Frage stellen. Einerseits haben die Ensemblemitglieder von Theater HORA durch Freie Republik HORA die Gelegenheit erhalten, sich in künstlerischer Selbstbestimmtheit und Eigenverantwortung zu üben. Andererseits haben Sie in den ersten drei Phasen viel mit Publikumsgesprächen und Tryouts gearbeitet - ausschließlich das Publikum und die an der Inszenierung beteiligten Schauspieler_innen durften Feedback zu den jeweiligen Inszenierungen geben, was zeigt, dass es in Freie Republik HORA auch um die Mündigkeit des Publikums geht. Die Diskussionen mit den Zuschauer_innen waren ein zentraler Aspekt für die Entwicklung der Inszenierungen. Wie wird dieser Aspekt nun in dieser vierten Phase mit Gott verhandelt? Welche Rolle spielt das Publikum?

Nele Jahnke: In den ersten drei Phasen haben wir alles transparent gemacht und sowohl die Meinung der Künstler_innen als auch diejenige des Publikums in den Fokus gestellt, wobei die künstlerischen Ansichten von Michael Elber und mir außen vor gelassen wurden. Für die vierte Phase haben wir uns darauf geeinigt, dass auf die Phasen der totalen Transparenz die Phase der absoluten Vollverschleierung folgen soll. Bei Gott sieht man nicht, wie das Stück erarbeitet wurde und wer die Verantwortung trägt. Da dem Publikum die in den Tryouts der zweiten Phase zugesprochene aktive Kritik-Instanz in dieser vierten Phase nicht mehr zusteht, wird es entmachtet. Wir wollen uns auf unsere künstlerischen Wünsche konzentrieren, anstatt uns an den Äußerungen der Zuschauenden zu orientieren.

Diskussionsteilnehmer_in: Meine Frage schließt unmittelbar an das Thema der erwähnten Vollverschleierung an. Meine Zuschauererfahrung gestern bestand vor allem darin, förmlich darunter zu leiden, immobilisiert zu sein und mich nicht im Bühnenraum bewegen zu können. Auf der Bühne herrschte eine Art Workshop-Dynamik. Ich saß so weit weg von Ihnen, die Sie auf der Bühne standen, und ich hatte das Bedürfnis, näher bei Ihnen zu sein und diesen gemeinsamen 
Raum mit Ihnen zu teilen. Aufgrund dieser Erfahrung wollte ich Sie fragen, ob Sie im Rahmen dieses Projekts Freie Republik HORA auch vorhaben, über Publikumsgespräche hinaus mit Formen zu arbeiten, bei welchen Sie das Publikum stärker miteinbeziehen?

Nele Jahnke: Das ist eine interessante Frage. Bis jetzt war eine solche Form noch nicht vorgesehen. In den vorherigen Phasen ging es immer ganz stark um den Austausch mit dem Publikum und um die Frage, wie wir über das, was wir sehen, sprechen. Gleichzeitig war ein wichtiger Punkt aber auch die Frage, wie die regieführende Person mit künstlerischen Äußerungen oder Kritik umgeht. Es ging im Grunde um die Auseinandersetzung zwischen Künstler_innen und Publikum. Unsere Erfahrung mit Publikumsgesprächen war dann jedoch, dass sich selten jemand wirklich getraut hat, etwas zu fragen. Die Kommunikation erwies sich als schwierig und zeitintensiv, da gestellte Fragen so umformuliert werden mussten, dass alle sie verstehen. Aus dieser Erfahrung heraus entwickelte sich unser Wunsch nach anderen Dialogen oder auch nach anderen Begriffen. Vielleicht wäre ein möglicher nächster Schritt, das Publikum stärker miteinzubeziehen. Wir müssen zudem überlegen, wie der Eindruck des >Labors` entsteht und wohin wir zusammen gehen wollen. Ich weiß es noch nicht und die anderen Ensemblemitglieder wahrscheinlich auch nicht.

Diskussionsteilnehmer_in: Herr Blumer, wenn Sie vorne auf der Bühne stehen, denken Sie dann darüber nach, was ihr Spiel beim Publikum auslösen könnte?

Gianni Blumer: Wenn ich vorne auf der Bühne stehe, fühle ich mich eigentlich immer wohl mit dem Publikum und ich habe kein Lampenfieber, nein! Ich zeige einfach mein Talent. Dieses Talent entsteht aus meiner Beeinträchtigung, die ich habe.

Diskussionsteilnehmer_in: Herr Blumer, mir fällt auf, dass, wenn Sie auf der Bühne stehen, Sie viel mit dem Publikum kommunizieren. Beispielsweise blicken und lachen Sie die Zuschauenden an. In der 
Wissenschaft nennen wir das ein Durchbrechen der Vierten Wand, womit Sie, Herr Blumer, ganz stark spielen. Machen Sie das gerne?

Gianni Blumer: Das ist halt so. Ich möchte dem Publikum gefallen. Auch habe ich noch mehr Ideen, was ich mit dem Publikum machen könnte. Zum Beispiel würde ich gerne Querflöte spielen und mit hübschen jungen Frauen tanzen. Das mache ich am liebsten. Deswegen halte ich das Publikum meistens auf Trab, weil ich auch den Mut dafür habe. Und mir gefällt das einfach, das Publikum mit in die Gruppe zu nehmen.

\section{Anmerkungen}

1 Das Projekt Freie Republik HORA verfolgt zwei zentrale Ziele. Einerseits sollen die Künstler_innen von Theater HORA zum ersten Mal als Regisseur_innen Autorschaft erhalten und sich in künstlerischer Autonomie üben. Andererseits geht es um die Mündigkeit des Publikums, das als einzige Instanz dazu befugt ist, Kritik an den Regiearbeiten zu äußern. Es stellte sich jedoch heraus, dass es für die Zuschauenden eine Herausforderung darstellt, die individuellen Meinungen gegenüber den Ensemblemitglieder zu äußern, weswegen neue Formate von Publikumsgesprächen entwickelt wurden, die spielerische Formen aufwiesen und nicht nur auf einem sprachlichen Austausch basierten. Folglich werden die beiden Ziele von Freie Republik HORA im Rahmen von sogenannten Tryouts verhandelt, womit sie als Laboratorien angesehen werden können, in denen der Austausch zwischen Künstler_innen und Publikum erprobt wird.

2 Im Rahmen des AUAWIRLEBEN Theaterfestival Bern wurde die Vorstellung von Gott vom 19.5.2017 im Tojo Theater in der Reitschule in Bern besucht.

3 Das laborartige Stück Gott ist bereits im Gange, wenn die Zuschauer_innen den Raum betreten. Gleich zu Beginn wird die Selbstermächtigung der einzelnen Performer_innen und die nicht-hierarchische Konstellation des Abends deutlich gemacht. Die am Bühnenrand sitzendenden und dadurch einen Halbkreis formenden Performer_innen stehen nacheinander auf, jede_r Einzelne stellt sich als Regisseur_in vor und erklärt, was sie beziehungsweise er sich wünscht. In einem weiteren Schritt wird die eigentliche Machtfigur und Thematik des Abends eingeführt: „Gott«. 
(c) by Alexander Verlag Berlin 2018

Alexander Wewerka, Postfach 1818 24, 14008 Berlin info@alexander-verlag.com | www.alexander-verlag.com Alle Rechte vorbehalten. Jede Form der Vervielfältigung, auch der auszugsweisen, nur mit Genehmigung des Verlags.

Die vorliegende elektronische Version wurde auf Bern Open Publishing (http://bop.unibe.ch/itwid) publiziert. Es gilt die Lizenz Creative Commons Namensnennung - Weitergabe unter gleichen Bedingungen, Version 4.o (CC BY-SA 4.0). Der Lizenztext ist einsehbar unter: http://creativecommons.org/licenses/by-sa/4.o/deed.de

ISBN (Druckversion): 978-3-89581-478-5

ISBN (elektronische Version): 978-3-89581-506-5 